\title{
IMRT Beam Angle Optimization Using Non-Descent Pattern Search Methods
}

\author{
Humberto Rocha ${ }^{1}$, Joana M. Dias ${ }^{1,2}$, Brigida C. Ferreira ${ }^{3,4}$, and \\ Maria do Carmo Lopes ${ }^{3,4}$ \\ 1 INESC-Coimbra, Rua Antero de Quental, 199 \\ 3000-033 Coimbra, Portugal \\ 2 Faculdade de Economia, Universidade de Coimbra, \\ 3004-512 Coimbra, Portugal \\ 3 I3N, Departamento de Física, Universidade de Aveiro, \\ 3810-193 Aveiro, Portugal \\ 4 Serviço de Física Médica, IPOC-FG, EPE, \\ 3000-075 Coimbra, Portugal \\ hrocha@mat.uc.pt, joana@fe.uc.pt, brigida@ua.pt, \\ mclopes@ipocoimbra.min-saude.pt
}

\begin{abstract}
The intensity-modulated radiation therapy (IMRT) treatment planning is usually a sequential process where initially a given number of beam directions are selected followed by the fluence map optimization (FMO) considering those beam directions. The beam angle optimization (BAO) problem consists on the selection of appropriate radiation incidence directions in radiation therapy treatment planning and may be decisive for the quality of the treatment plan, both for appropriate tumor coverage and for enhancement of better organs sparing. This selection must be based on the optimal value of the FMO problem otherwise the resulting beam angle set has no guarantee of optimality and has questionable reliability. Pattern search methods (PSM) have been used successfully to address the BAO problem driven by the optimal fluence value of the FMO problem. PSM are iterative methods generating a sequence of non-increasing iterates such that iterate progression is solely based on a finite number of function evaluations in each iteration, without explicit or implicit use of derivatives. Typically, in IMRT optimization, the quality of the solutions obtained is not simply related to the final value of an objective function but rather judged by dosevolume histograms or considering a set of physical dose metrics. These dose metrics can be simply described as obtaining a minimum prescribed dose for the target volumes (the regions that have to be irradiated) and a maximum or mean tolerance dose values for the remaining surrounding structures (the regions that should be spared). The goal of this paper is to present a non-descent PSM that can be guided both by an objective function formulation of the FMO problem and by physical dose metrics. Four retrospective treated cases of head-and-neck tumors at the Portuguese Institute of Oncology of Coimbra are used to discuss the benefits of non-descent PSM for the optimization of the BAO problem.
\end{abstract}

Keywords: Pattern Search Methods, IMRT, Beam Angle Optimization 


\section{Introduction}

Radiation therapy is with surgery and chemotherapy one of the three main treatment approaches for cancer, used for around $50 \%$ of all patients. With this modality, patients are irradiated with beams of ionizing radiation attempting to sterilize all cancer cells while minimizing the collateral effects on the surrounding healthy organs and tissues. An important type of radiation therapy is intensitymodulated radiation therapy (IMRT), a modern technique where the radiation beam is modulated by a multileaf collimator allowing the irradiation of the patient using non-uniform radiation fields from selected angles. The ionizing radiation is generated by a linear accelerator mounted on a gantry that can rotate along a central axis and is delivered with the patient immobilized on a couch that can rotate. The rotation of the couch combined with the rotation of the gantry allows radiation from almost any angle around the tumor. Despite that fact, the use of angles that lay in the plane of rotation of the gantry, i.e. coplanar angles, is predominant. The selection of appropriate radiation incidence directions in radiation therapy treatment planning - beam angle optimization (BAO) problem - is important for the quality of the treatment plan [5,13], both for appropriate tumor coverage and for better organ sparing. However, in clinical practice, the beam angle number and directions are typically selected in a timeconsuming trial-and-error procedure by a dosimetrist. The fact that the BAO problem is a highly non-convex optimization problem with many local minima [4], yet to be solved in a satisfactory way within a clinically acceptable time frame, helps explaining the current clinical practice.

The BAO problem is the first problem that arises in treatment planning, but its optimal solution is highly dependent on the optimal solution of the fluence map optimization (FMO) problem - the problem of deciding what are the optimal radiation intensities associated with each set of beam angles. When the BAO problem is not based on the optimal FMO solutions, the resulting beam angle set has no guarantee of optimality and has questionable reliability since it has been extensively reported that optimal beam angles for IMRT are often non-intuitive [21]. Obtaining the optimal solution for a beam angle set is time costly and even if only one beam angle is changed in that set, it is necessary to calculate the radiation dose that is being deposited in the patient's tissues - organs and tumors. Therefore, methods that avoid being easily trapped in local minima and that require few function value evaluations to progress and converge are advantageous. The pattern search methods (PSM) framework has been used by us to address the BAO problem successfully due to its ability to avoid local entrapment and its need for few function value evaluations to converge $[16,17,18,19]$.

PSM are iterative methods generating a sequence of non-increasing iterates such that iterate progression is solely based on a finite number of function evaluations in each iteration, without explicit or implicit use of derivatives. In IMRT, the final value of an objective function is not a complete and unique measure of the quality of the solution obtained. In clinical practice, the quality of the solution obtained is rather judged by dose-volume histograms or considering a set of physical dose metrics. These dose metrics can be simply described as ob- 
taining a minimum prescribed dose for the target volumes (the regions that have to be irradiated) and a maximum or mean tolerance dose values for the remaining surrounding structures (the regions that should be spared). The goal of this paper is to present a non-descent PSM that can be guided both by the objective function of the FMO problem and by the dose metrics. Typically, the progression of PSM is determined by the decrease in the objective function value. In this paper, small increases of the objective function value are allowed whenever some physical feature of the problem is improved at the cost of other feature(s) that still remain within the limit(s) prescribed. Four retrospective treated cases of head-and-neck tumors at the Portuguese Institute of Oncology of Coimbra are used to discuss the benefits of non-descent PSM for the optimization of the $\mathrm{BAO}$ problem. The paper is organized as follows. In the next section we describe the BAO problem. Non-descent PSM framework is presented in section 3. Computational tests using clinical examples of head-and-neck cases are presented in section 4. In the last Section we have the conclusions.

\section{Beam Angle Optimization in IMRT Treatment Planning}

The BAO problem is a quite difficult problem to solve since it is a highly non-convex optimization problem with many local minima - see Fig. 1. In most of the previous works on $\mathrm{BAO}$, the entire range $\left[0^{\circ}, 360^{\circ}\right]$ of gantry angles is discretized into equally spaced beam directions with a given angle increment, such as 5 or 10 degrees, where exhaustive searches are performed directly or guided by a variety of different heuristics including simulated annealing [3], genetic algorithms [10], particle swarm optimization [12] or other heuristics incorporating a priori knowledge of the problem [15]. Although those global heuristics can theoretically avoid local optima, globally optimal or even clinically better solutions can not be obtained without a large number of objective function evaluations. On the other hand, the use of single-beam metrics has been a popular approach to address the BAO problem as well, e.g., the concept of beam's-eye-view [14]. Despite the computational time efficiency of these approaches, the quality of the solutions proposed cannot be guaranteed since the interplay between the selected beam directions is ignored.

In order to model the $\mathrm{BAO}$ problem as a mathematical programming problem, a quantitative measure to compare the quality of different sets of beam angles is required. For the reasons presented in Section 1, our approach for modeling the BAO problem uses the optimal solution value of the FMO problem as the measure of the quality for a given beam angle set. Thus, we will present the formulation of the BAO problem followed by the formulation of the FMO problem we used. Here, we will assume that the number of beam angles is defined a priori by the treatment planner and that all the radiation directions lie on the same plane. 

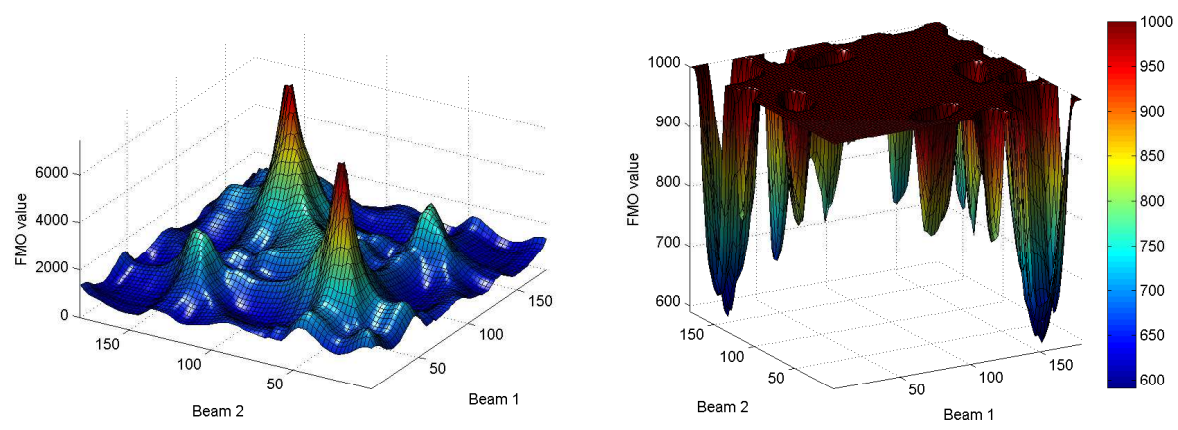

Fig. 1. 2-beam BAO surface (left) and truncated surface (right) to highlight the many local minima.

\subsection{BAO Model}

Let us consider $n$ to be the fixed number of (coplanar) beam directions, i.e., $n$ beam angles are chosen on a circle around the CT-slice of the body that contains the isocenter (usually the center of mass of the tumor). In our formulation, instead of a discretized sample, all continuous $\left[0^{\circ}, 360^{\circ}\right]$ gantry angles will be considered. Since the angle $-1^{\circ}$ is equivalent to the angle $359^{\circ}$ and the angle $361^{\circ}$ is the same as the angle $1^{\circ}$, we can avoid a bounded formulation. A simple formulation for the $\mathrm{BAO}$ problem is obtained by selecting an objective function such that the best set of beam angles is obtained for the function's minimum:

$$
\begin{aligned}
& \min f\left(\theta_{1}, \ldots, \theta_{n}\right) \\
& \text { s.t. }\left(\theta_{1}, \ldots, \theta_{n}\right) \in \mathbb{R}^{n} .
\end{aligned}
$$

Here, for the reasons stated before, the objective $f\left(\theta_{1}, \ldots, \theta_{n}\right)$ that measures the quality of the set of beam directions $\theta_{1}, \ldots, \theta_{n}$ is the optimal value of the FMO problem for each fixed set of beam directions. The FMO model used is presented next.

\section{$2.2 \quad$ FMO Model}

In order to solve the FMO problem, i.e., to determine optimal fluence maps, the radiation dose distribution deposited in the patient needs to be assessed accurately. Each structure's volume is discretized into small volume elements (voxels) and the dose is computed for each voxel considering the contribution of each beamlet. Typically, a dose matrix D is constructed from the collection of all beamlet weights, by indexing the rows of $\mathrm{D}$ to each voxel and the columns to each beamlet, i.e., the number of rows of matrix $\mathrm{D}$ equals the number of voxels $\left(N_{v}\right)$ and the number of columns equals the number of beamlets $\left(N_{b}\right)$ from all beam directions considered. Therefore, using matrix format, we can say that the 
total dose received by the voxel $i$ is given by $\sum_{j=1}^{N_{b}} D_{i j} w_{j}$, with $w_{j}$ the weight of beamlet $j$. Usually, the total number of voxels is large, reaching the tens of thousands, which originates large-scale problems. This is one of the main reasons for the difficulty of solving the FMO problem.

For a given beam angle set, an optimal IMRT plan is obtained by solving the FMO problem - the problem of determining the optimal beamlet weights for the fixed beam angles. Many mathematical optimization models and algorithms have been proposed for the FMO problem, including linear models [20], mixed integer linear models [11] and nonlinear models [2]. Here, we will use this later approach that penalizes each voxel according to the square difference of the amount of dose received by the voxel and the amount of dose desired/allowed for the voxel. This formulation yields a quadratic programming problem with only linear non-negativity constraints on the fluence values [20]:

$$
\begin{aligned}
& \min _{w} \sum_{i=1}^{N_{v}} \frac{1}{v_{S}}\left[\underline{\lambda}_{i}\left(T_{i}-\sum_{j=1}^{N_{b}} D_{i j} w_{j}\right)_{+}^{2}+\bar{\lambda}_{i}\left(\sum_{j=1}^{N_{b}} D_{i j} w_{j}-T_{i}\right)_{+}^{2}\right] \\
& \text { s.t. } \quad w_{j} \geq 0, j=1, \ldots, N_{b},
\end{aligned}
$$

where $T_{i}$ is the desired dose for voxel $i$ of the structure $v_{S}, \underline{\lambda}_{i}$ and $\bar{\lambda}_{i}$ are the penalty weights of underdose and overdose of voxel $i$, and $(\cdot)_{+}=\max \{0, \cdot\}$. This nonlinear formulation implies that a very small amount of underdose or overdose may be accepted in clinical decision making, but larger deviations from the desired/allowed doses are decreasingly tolerated [2].

The FMO model is used as a black-box function and the conclusions drawn regarding BAO coupled with this nonlinear model are valid also if different FMO formulations are considered.

\section{Non-descent Pattern Search Methods}

PSM framework will be briefly described followed by the presentation of the proposed non-descent PSM algorithm tailored for the BAO problem.

\subsection{Pattern Search Methods Framework}

PSM use the concept of positive bases (or positive spanning sets) to move towards a direction that would produce a function decrease. A positive basis for $\mathbb{R}^{n}$ can be defined as a set of nonzero vectors of $\mathbb{R}^{n}$ whose positive combinations span $\mathbb{R}^{n}$ (positive spanning set), but no proper set does. A positive spanning set contains at least one positive basis. It can be shown that a positive basis for $\mathbb{R}^{n}$ contains at least $n+1$ vectors and cannot contain more than $2 n$ [8]. Positive bases with $n+1$ and $2 n$ elements are referred to as minimal and maximal positive basis, respectively. Commonly used minimal and maximal positive bases are $[I-e]$, with $I$ being the identity matrix of dimension $n$ and $e=\left[\begin{array}{lll}1 & \ldots & 1\end{array}\right]^{\top}$, and $\left[\begin{array}{ll}I & -I\end{array}\right]$, 
respectively. The motivation for directional direct search methods such as PSM is given by one of the main features of positive basis (or positive spanning sets) [8]: there is always a vector $\mathbf{v}^{i}$ in a positive basis (or positive spanning set) that is a descent direction unless the current iterate is at a stationary point, i.e., there is an $\alpha>0$ such that $f\left(x^{k}+\alpha \mathbf{v}^{i}\right)<f\left(x^{k}\right)$. This is the core of directional direct search methods and in particular of PSM. The notions and motivations for the use of positive bases, its properties and examples can be found in $[1,8]$.

PSM are iterative methods generating a sequence of non-increasing iterates $\left\{x_{k}\right\}$. Given the current iterate $x^{k}$, at each iteration $k$, the next point $x^{k+1}$, aiming to provide a decrease of the objective function, is chosen from a finite number of candidates on a given mesh $M_{k}$ defined as

$$
M_{k}=\left\{x^{k}+\alpha_{k} \mathbf{V} \mathbf{z}: \mathbf{z} \in \mathbb{Z}_{+}^{p}\right\},
$$

where $\alpha_{k}$ is the mesh-size (or step-size) parameter, $\mathbb{Z}_{+}$is the set of nonnegative integers and $\mathbf{V}$ denote the $n \times p$ matrix whose columns correspond to the $p$ $(\geq n+1)$ vectors forming a positive spanning set.

PSM are organized around two steps at every iteration. The first step consists of a finite search on the mesh, free of rules, with the goal of finding a new iterate that decreases the value of the objective function at the current iterate. This step, called the search step, has the flexibility to use any strategy, method or heuristic, or take advantage of a priori knowledge of the problem at hand, as long as it searches only a finite number of points in the mesh. The search step provides the flexibility for a global search since it allows searches away from the neighborhood of the current iterate, and influences the quality of the local minimizer or stationary point found by the method.

If the search step fails to produce a decrease in the objective function, a second step, called the poll step, is performed around the current iterate. The poll step follows stricter rules and, using the concepts of positive bases, attempts to perform a local search in a mesh neighborhood around $\mathbf{x}^{k}, \mathcal{N}\left(\mathbf{x}^{k}\right)=\left\{\mathbf{x}^{k}+\right.$ $\alpha_{k} \mathbf{v}$ : for all $\left.\mathbf{v} \in P_{k}\right\} \subset M_{k}$, where $P_{k}$ is a positive basis chosen from the finite positive spanning set $\mathbf{V}$. For a sufficiently small mesh-size parameter $\alpha_{k}$, the poll step is guaranteed to provide a function reduction, unless the current iterate is at a stationary point [1]. So, if the poll step also fails to produce a function reduction, the mesh-size parameter $\alpha_{k}$ must be decreased. On the other hand, if both the search and poll steps fail to obtain an improved value for the objective function, the mesh-size parameter is increased or held constant. The most common choice for the mesh-size parameter update is to halve the mesh-size parameter at unsuccessful iterations and to keep it or double it at successful ones. The PSM framework is summarized in Algorithm 1.

\subsection{Non-descent Pattern Search Methods for BAO}

PSM are derivative-free optimization algorithms widely used for the minimization of non-convex functions such that iterate progression is solely based on a finite number of function evaluations in each iteration, without explicit 


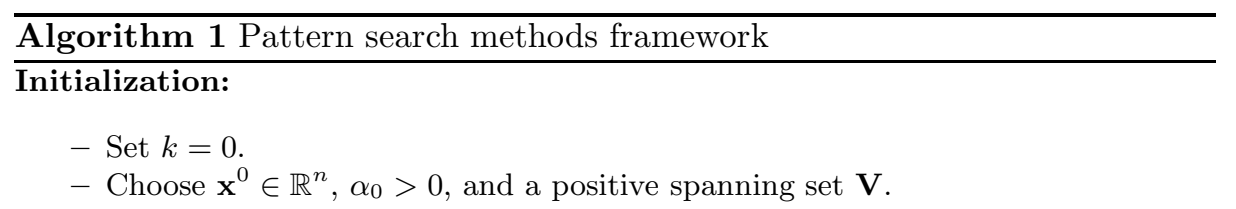

\section{Iteration:}

1. Search step: evaluate $f$ at a finite number of points in $M_{k}$ with the goal of decreasing the objective function value at $\mathbf{x}^{k}$. If $\mathbf{x}^{k+1} \in M_{k}$ is found satisfying $f\left(\mathbf{x}^{k+1}\right)<f\left(\mathbf{x}^{k}\right)$, go to step 4 and expand $M_{k}$. Both search step and iteration are declared successful. Otherwise, go to step 2 and search step is declared unsuccessful.

2. Poll step: this step is only performed if the search step is unsuccessful. If $f\left(\mathbf{x}^{k}\right) \leq$ $f(\mathbf{x})$ for every $\mathbf{x}$ in the mesh neighborhood $\mathcal{N}\left(\mathbf{x}^{k}\right)$, go to step 3 and shrink $M_{k}$. Both poll step and iteration are declared unsuccessful. Otherwise, choose a point $\mathbf{x}^{k+1} \in \mathcal{N}\left(\mathbf{x}^{k}\right)$ such that $f\left(\mathbf{x}^{k+1}\right)<f\left(\mathbf{x}^{k}\right)$, go to step 4 and expand $M_{k}$. Both poll step and iteration are declared successful.

3. Mesh reduction: let $\alpha_{k+1}=\frac{1}{2} \times \alpha_{k}$. Set $k \leftarrow k+1$ and return to step 1 for a new iteration.

4. Mesh expansion: let $\alpha_{k+1}=\alpha_{k}$ (or $\alpha_{k+1}=2 \times \alpha_{k}$ ). Set $k \leftarrow k+1$ and return to step 1 for a new iteration.

or implicit use of derivatives. In IMRT, the objective function value is not a unique measure of the quality of a given solution. For the assessment of the clinical expected outcome, dose-volume histograms and a set of physical dose metrics should be considered. The tumor to be treated plus some safety margins is called planning target volume (PTV). For PTV, an important dose metric is the volume of PTV that receives $95 \%$ of the prescribed dose. Typically, $95 \%$ of the PTV volume is required. Mean and/or maximum doses are usually the most important dose metrics for the organ's at risk (OARs). There are many different ways to incorporate these dose metrics in a flexible PSM framework. They can be used within the poll step to decide which point to choose when more than one point improves the best objective function value. They can be used to accept a trial point if it improves the best objective function value and also improves the dose metrics. Our strategy, based on extensive numerical experiments, is to accept a trial point if it improves the best objective function value or if it improves the dose metrics and the objective function value is within a radius of the best objective function value. This proposal considers that the choice of beam angle sets with similar objective function value of the current beam angle set, i.e. $f\left(x^{k+1}\right)<f\left(x^{k}\right)+\epsilon$, should be made using dose metrics, directing the algorithm to regions of the search space where better dose metrics are obtained.

Deciding which set of beam angles have the best dose metrics is not straightforward. For the PTV we consider the dose metric fulfilled if $95 \%$ of the PTV volume receives more than $95 \%$ of the prescribed dose. For an OAR the dose metric is fulfilled if the maximum dose (or the mean dose depending on the type 
of organ) is under the prescribed values. In the context of the BAO process, we consider that a beam angle set improves the dose metrics of the current beam angle set when all the dose metrics already fulfilled remain fulfilled and one or more dose metrics yet to be fulfilled are improved. For example, for a current beam angle set that satisfies dose metrics for all structures except one OAR, a beam angle set is considered as improving the dose metrics if it improves the dose metric of the OAR yet to be fulfilled and all the other structure's dose metrics remain fulfilled. Necessarily some of the already fulfilled dose metrics will be deteriorated but the prescribed dose limits will continue to be assured. When and while all structures have dose metrics fulfilled, the BAO process progression is only determined by decreases on the objective function value, meaning that structures considered more important in the objective function value will be better spared/irradiated. The strategy adopted here attempts to maximize the number of structures with the dose metrics fulfilled which roughly correspond to fulfill the prescribed doses by the medical doctor. It should be highlighted that, in our tests, most of the times, we could not obtain a treatment plan that fulfill the prescribed doses for all structures. The strategy sketched for non-descent PSM to address the BAO problem is presented in Algorithm 2.

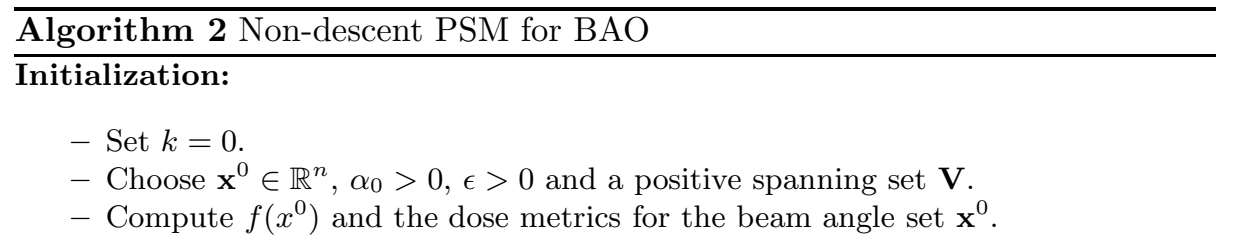

\section{Iteration:}

1. Search step: evaluate $f$ at a finite number of points in $M_{k}$ with the goal of improving the current beam angle set. If $x^{k+1} \in M_{k}$ is found satisfying $f\left(x^{k+1}\right)<$ $f\left(x^{k}\right)$ or $f\left(x^{k+1}\right)<f\left(x^{k}\right)+\epsilon$ and the dose metrics of $x^{k+1}$ improve the dose metrics of $x^{k}$, go to step 4, expand $M_{k}$ and compute the dose metrics of the new best beam angle set. Both search step and iteration are declared successful. Otherwise, go to step 2 and search step is declared unsuccessful.

2. Poll step: this step is only performed if the search step is unsuccessful. If there is a point $x^{k+1} \in \mathcal{N}\left(x^{k}\right)$ such that $f\left(x^{k+1}\right)<f\left(x^{k}\right)$ or $f\left(x^{k+1}\right)<f\left(x^{k}\right)+\epsilon$ and the metrics of $x^{k+1}$ improve the metrics of $x^{k}$, both poll step and iteration are declared successful, go to step 4 and compute the dose metrics of the new best beam angle set. Otherwise, if every $x$ in the mesh neighborhood $\mathcal{N}\left(x^{k}\right)$, fail to improve the current beam angle set then go to step 3 and shrink $M_{k}$. Both poll step and iteration are declared unsuccessful.

3. Mesh reduction: let $\alpha_{k+1}=\frac{1}{2} \times \alpha_{k}$. Set $k \leftarrow k+1$ and return to step 1 for a new iteration.

4. Mesh expansion: let $\alpha_{k+1}=\alpha_{k}$ (or $\alpha_{k+1}=2 \times \alpha_{k}$ ). Set $k \leftarrow k+1$ and return to step 1 for a new iteration. 
The efficiency of PSM improved significantly by reordering the poll directions according to descent indicators built from simplex gradients [7]. Adding to the efficiency provided by an insightful reordering of the poll directions, the search step was recently provided with the use of minimum Frobenius norm quadratic models to be minimized within a trust region, which can lead to a significant improvement of direct search for smooth, piecewise smooth, and noisy problems [6]. For implementation and comparison of the non-descent PSM algorithm for the BAO problem, we use as basis the last version of SID-PSM $[6,7]$ which is a MATLAB implementation of the PSM.

\section{Computational Results for Head-and-Neck Clinical Examples}

The non-descent PSM algorithm was tested using four clinical examples of retrospective treated cases of head-and-neck tumors at the Portuguese Institute of Oncology of Coimbra (IPOC). In general, the head-and-neck region is a complex area to treat with radiotherapy due to the large number of sensitive organs in this region (e.g., eyes, mandible, larynx, oral cavity, etc.). For simplicity, in this study, the OARs used for treatment optimization were limited to the spinal cord, the brainstem and the parotid glands. The spinal cord and the brainstem are some of the most critical OARs in the head-and-neck tumor cases. These are serial organs, i.e., organs such that if only one subunit is damaged, the whole organ functionality is compromised. Therefore, if the tolerance dose is exceeded, it may result in functional damage to the whole organ. Thus, it is extremely important not to exceed the tolerance dose prescribed for these type of organs. Other than the spinal cord and the brainstem, the parotid glands are also important OARs. The parotid gland is the largest of the three salivary glands. A common complication due to parotid glands irradiation is xerostomia (the medical term for dry mouth due to lack of saliva). This decreases the quality of life of patients undergoing radiation therapy of head-and-neck, causing difficulties to swallow. The parotids are parallel organs, i.e., if a small volume of the organ is damaged, the rest of the organ functionality may not be affected. Their tolerance dose depends strongly on the fraction of the volume irradiated. Hence, if only a small fraction of the organ is irradiated the tolerance dose is much higher than if a larger fraction is irradiated. Thus, for these parallel structures, the organ mean dose is generally used instead of the maximum dose as an objective for inverse planning optimization. For the head-and-neck cases in study, PTV was separated in two parts with different prescribed doses: PTV1 and PTV2. The prescription dose for the target volumes and tolerance doses for the OARs considered in the optimization are presented in Table 1.

Our tests were performed on a 2.66Ghz Intel Core Duo PC with 3 GB RAM. The patients' CT sets and delineated structures are exported via Dicom RT to a freeware computational environment for radiotherapy research - CERR [9]. We used CERR 3.2.2 version and MATLAB 7.4.0 (R2007a). An automatized procedure for dose computation for each given beam angle set was developed, 
Table 1. Prescribed doses for all the structures considered for IMRT optimization.

\begin{tabular}{lccc}
\hline Structure & Mean dose Max dose Prescribed dose \\
\hline Spinal cord & - & $45 \mathrm{~Gy}$ & - \\
Brainstem & - & $54 \mathrm{~Gy}$ & - \\
Left parotid & $26 \mathrm{~Gy}$ & - & - \\
Right parotid & $26 \mathrm{~Gy}$ & - & - \\
PTV1 & - & - & $70.0 \mathrm{~Gy}$ \\
PTV2 & - & - & $59.4 \mathrm{~Gy}$ \\
Body & - & $80 \mathrm{~Gy}$ & - \\
\hline
\end{tabular}

instead of the traditional dose computation available from IMRTP module accessible from CERR's menubar. This automatization of the dose computation was essential for integration in our BAO algorithm. To address the convex nonlinear formulation of the FMO problem we used a trust-region-reflective algorithm (fmincon) of MATLAB 7.4.0 (R2007a) Optimization Toolbox.

We choose to implement the non-descent PSM algorithm taking advantage of the availability of an existing PSM framework implementation used successfully by us to tackle the BAO problem $[16,17,18,19]$ - the last version of SID-PSM $[6,7]$. The spanning set used was the positive spanning set $\left(\left[\begin{array}{lll}e-e & I-I\end{array}\right]\right.$. Each of these directions corresponds to, respectively, the rotation of all incidence directions clockwise, the rotation of all incidence directions counter-clockwise, the rotation of each individual incidence direction clockwise, and the rotation of each individual incidence direction counter-clockwise.

Treatment plans with five to nine equispaced coplanar beams are used at IPOC and are commonly used in practice to treat head-and-neck cases [2]. We considered plans with five coplanar beams because the importance of BAO increases when a lower number of beam directions is considered. Therefore, treatment plans of five coplanar orientations were obtained using SID-PSM and using non-descent PSM algorithm denoted ND-PSM. These plans were compared with the typical 5-beam equispaced coplanar treatment plans denoted equi. Since we want to improve the quality of the typical equispaced treatment plans, the starting point considered is the equispaced coplanar beam angle set.

The history of the BAO comparing the objective function value decrease versus the number of function evaluations for the four clinical cases of head-andneck tumors using $S I D-P S M$ and $N D-P S M$ are displayed in Fig. 2. Due to its non-descent nature, ND-PSM required a larger number of function evaluations to converge. However, most of the times, it lead to better results in terms of objective function value obtained. The main purpose of the strategy delineated for the non-descent PSM algorithm was to obtain solutions with better dose metrics results regardless of the final objective function obtained. Nevertheless, the strategy of directing the search to neighborhoods with better dose metrics apparently also benefits the obtention of improvements in the final objective function value. 


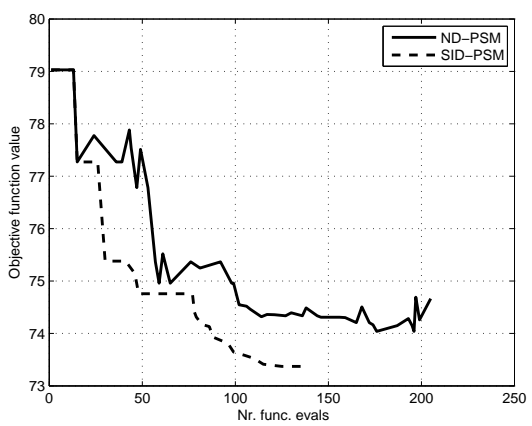

(a)

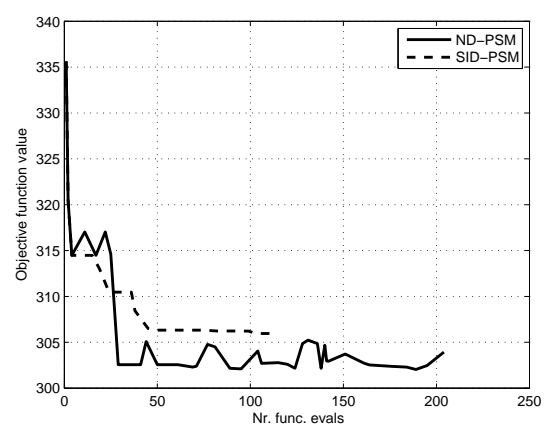

(c)

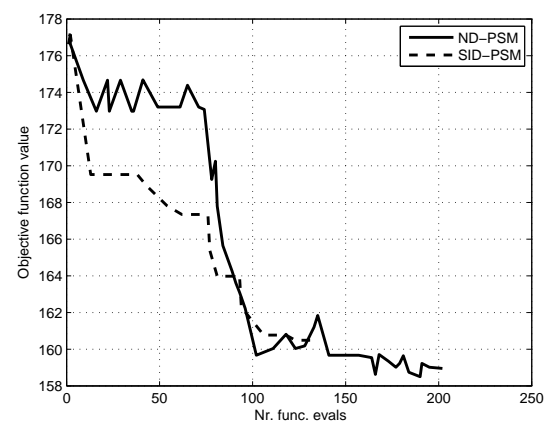

(b)

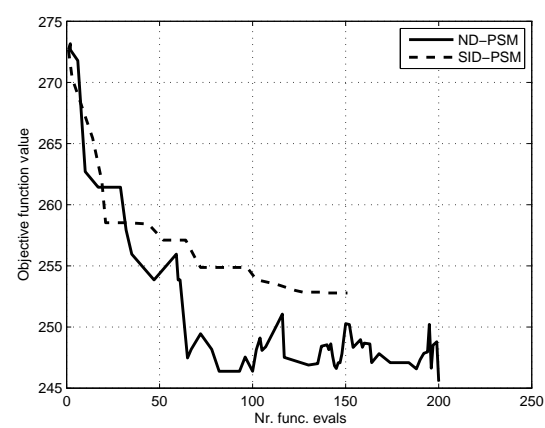

(d)

Fig. 2. History of the 5-beam angle optimization process using $S I D-P S M$ and $N D$ $P S M$, considering the equispaced configuration (equi) as starting point, for cases 1 to 4,2 (a) to $2(\mathrm{~d})$ respectively.

Despite the improvement in FMO value, as referred previously, the quality of the results can be perceived considering a variety of metrics. A metric usually used for plan evaluation is the volume of PTV that receives $95 \%$ of the prescribed dose. Typically, $95 \%$ of the PTV volume is required. The occurrence of coldspots, less than $93 \%$ of PTV volume receives the prescribed dose, and the existence of hotspots, the percentage of the PTV volume that receives more than $110 \%$ of the prescribed dose, are other measures usually used to evaluate target coverage. Mean and/or maximum doses of OARs are usually displayed to verify organ sparing.

The results regarding targets coverage are presented in Table 2. Using only 5 beam directions makes harder to obtain a satisfactory target coverage. We can verify that optimized treatment plans consistently obtained slightly better target coverage numbers compared to equi treatment plans. On the other hand, target coverage numbers are favorable to ND-PSM treatment plans compared to SID- 
Table 2. Target coverage obtained by treatment plans.

\begin{tabular}{|c|c|c|c|}
\hline Case & Target coverage & ND-PSM SID-PSM & I equi \\
\hline \multirow{6}{*}{1} & $\Gamma V 1$ at $95 \%$ & 67.07 Gy 67.32 Gy & $67.22 \mathrm{~Gy}$ \\
\hline & PTV1 $\%>93$ & 99.32 & 99.39 \\
\hline & PTV $1 \%>110 \%$ of $\mathrm{Rx}(\%)$ & 0.00 & 0.00 \\
\hline & PTV2 at $95 \%$ volume & $56.82 \mathrm{~Gy} 56.12 \mathrm{~Gy}$ & $55.47 \mathrm{~Gy}$ \\
\hline & PTV2 $\%>93 \%$ of $\mathrm{Rx}(\%)$ & 96.93 & 95.26 \\
\hline & PTV2 $\%>110 \%$ of $\mathrm{Rx}(\%)$ & 6.07 & 6.24 \\
\hline \multirow{6}{*}{2} & PTV1 at $95 \%$ volume & 65.77 Gy 65.07 Gy & 64.57 Gy \\
\hline & PTV1 $\%>93 \%$ of $\mathrm{Rx}(\%)$ & 95.18 & 93 \\
\hline & PTV $1 \%>110 \%$ of $\mathrm{Rx}(\%)$ & 0.00 & 0.02 \\
\hline & PTV2 at $95 \%$ volume & 56.57 Gy 56.72 Gy & $56.32 \mathrm{~Gy}$ \\
\hline & PTV2 $\%>9$ & 96.59 & 96.16 \\
\hline & PTV2 $\%>110 \%$ of $\mathrm{Rx}(\%)$ & 24.88 & 25.03 \\
\hline \multirow{6}{*}{3} & PTV1 at $95 \%$ volume & 67.27 Gy 66.97 Gy & $66.97 \mathrm{~Gy}$ \\
\hline & PTV1 $\%>93 \%$ & 99.21 & 99.21 \\
\hline & PTV $1 \%>110 \%$ of $\mathrm{Rx}(\%)$ & 0.00 & 0.00 \\
\hline & PTV2 at $95 \%$ volume & 55.02 Gy 55.07 Gy & $54.17 \mathrm{~Gy}$ \\
\hline & PTV2 \% 冫 & 94.88 & \\
\hline & PTV2 $\%>110 \%$ of $\mathrm{Rx}(\%)$ & 12.43 & 12.16 \\
\hline \multirow{6}{*}{4} & PTV1 at $95 \%$ volume & 65.27 Gy 64.97 Gy & $64.42 \mathrm{~Gy}$ \\
\hline & PTV1 $\%>93 \%$ of $\mathrm{Rx}(\%)$ & 95.23 & 93.93 \\
\hline & PTV $1 \%>110 \%$ of $\mathrm{Rx}(\%)$ & 0.00 & 0.00 \\
\hline & PTV2 at $95 \%$ volume & 58.07 Gy $58.22 \mathrm{~Gy}$ & $58.22 \mathrm{~Gy}$ \\
\hline & PTV2 \% & 98.61 & 98.20 \\
\hline & PTV2 \% > & 37.15 & 37.64 \\
\hline
\end{tabular}

PSM treatment plans, particularly when $95 \%$ of the prescribed dose of PTV1 (66.5 Gy) or PTV2 (56.43 Gy) is not fulfilled. Organ sparing results are shown in Table 3. All the treatment plans fulfill the maximum dose requirements for the spinal cord and the brainstem. However, as expected, the main differences reside in parotid sparing. The optimized treatment plans clearly improve the usually clinically used equispaced treatment plans. The equi treatment plans could never fulfill parotid sparing while ND-PSM treatment plans always fulfill the parotid's mean dose requirements except for the right parotid in case 3 . Although SID-PSM treatment plans reduced the parotid's mean dose as well, it does not manage to fulfill the dose limits prescribed as many times as $N D$ $P S M$ treatment plans. Curiously, despite the final objective function value being worse for $N D-P S M$, case 1 illustrates the benefits of the non-descent strategy with respect to parotid sparing. 
Table 3. OARs sparing obtained by treatment plans.

\begin{tabular}{|c|c|c|c|c|c|c|c|}
\hline \multirow[b]{2}{*}{ Case } & \multirow[b]{2}{*}{ OAR } & \multicolumn{3}{|c|}{ Mean Dose (Gy) } & \multicolumn{3}{|c|}{ Max Dose (Gy) } \\
\hline & & $N D-P S M$ & $S I D-P S M$ & equi & $N D-P S M$ & $S I D-P S M$ & equi \\
\hline \multirow{4}{*}{1} & Spinal cord & - & - & - & 44.10 & 40.05 & 41.32 \\
\hline & Brainstem & - & - & - & 53.18 & 51.90 & 51.86 \\
\hline & Left parotid & 25.85 & 25.81 & 26.63 & - & - & - \\
\hline & Right parotid & 24.68 & 26.60 & 26.32 & - & - & - \\
\hline \multirow{4}{*}{2} & Spinal cord & - & - & - & 39.59 & 41.26 & 40.30 \\
\hline & Brainstem & - & - & - & 43.78 & 40.34 & 39.49 \\
\hline & Left parotid & 25.72 & 26.31 & 26.58 & - & - & - \\
\hline & Right parotid & 25.11 & 24.34 & 26.45 & - & - & - \\
\hline \multirow{4}{*}{3} & Spinal cord & - & $\overline{-}$ & $\overline{-}$ & 37.96 & 37.94 & 38.10 \\
\hline & Brainstem & - & - & - & 50.43 & 50.68 & 50.20 \\
\hline & Left parotid & 24.43 & 24.37 & 27.02 & - & - & - \\
\hline & Right parotid & 26.74 & 29.00 & 29.44 & - & - & - \\
\hline \multirow{4}{*}{4} & Spinal cord & - & $\overline{-}$ & - & 39.37 & 40.14 & 38.68 \\
\hline & Brainstem & - & - & - & 52.94 & 52.12 & 52.26 \\
\hline & Left parotid & 22.29 & 23.32 & 26.86 & - & - & - \\
\hline & Right parotid & 21.39 & 24.07 & 26.96 & - & - & - \\
\hline
\end{tabular}

\section{Conclusions}

The ultimate goal of treatment planning is to be able to obtain a treatment plan that is in accordance with the medical prescription in terms of radiation dose distribution. Usually, the medical prescription will define prescribed doses to the target volumes, and mean or maximum tolerance doses to the organs at risk. This paper proposes an alternative approach to the BAO problem whose optimization process is simultaneously guided by dose metric goals and by an objective fucntion value. The PSM framework had already proved to be a suitable approach for the resolution of the non-convex BAO problem. For the clinical cases retrospectively tested, the use of dose metrics as decision criteria in our tailored approach showed a positive influence on the quality of the local minimizer found. The improvement of the solutions for the head-and-neck cases tested lead to high quality treatment plans with better target coverage and with improved organ sparing.

\section{Acknowledgements}

This work was supported by QREN under Mais Centro (CENTRO-07-0224FEDER-002003) and FEDER funds through the COMPETE program and Portuguese funds through FCT under project grant PTDC/EIA-CCO/121450/2010. This work has also been partially supported by FCT under project grant PEstOE/EEI/UI308/2014. The work of H. Rocha was supported by the European social fund and Portuguese funds from MCTES. 


\section{References}

1. Alberto, P., Nogueira, F., Rocha, H., Vicente, L.N.: Pattern search methods for user-provided points: Application to molecular geometry problems. SIAM J. Optim. 14, 1216-1236 (2004)

2. Aleman, D.M., Kumar, A., Ahuja, R.K., Romeijn, H.E., Dempsey, J.F.: Neighborhood search approaches to beam orientation optimization in intensity modulated radiation therapy treatment planning. J. Global Optim. 42, 587-607 (2008)

3. Bortfeld, T., Schlegel, W.: Optimization of beam orientations in radiation therapy: some theoretical considerations. Phys. Med. Biol. 38, 291-304 (1993)

4. Craft, D.: Local beam angle optimization with linear programming and gradient search. Phys. Med. Biol. 52, 127-135 (2007)

5. Das, S.K., Marks, L.B.: Selection of coplanar or non coplanar beams using threedimensional optimization based on maximum beam separation and minimized nontarget irradiation. Int. J. Radiat. Oncol. Biol. Phys. 38, 643-655 (1997)

6. Custódio, A.L., Rocha, H., Vicente, L.N.: Incorporating minimum Frobenius norm models in direct search. Comput. Optim. Appl. 46, 265-278 (2010)

7. Custódio, A.L., Vicente, L.N.: Using sampling and simplex derivatives in pattern search methods. SIAM J. Optim. 18, 537-555 (2007)

8. Davis, C.: Theory of positive linear dependence. Am. J. Math. 76, 733-746 (1954)

9. Deasy, J.O., Blanco, A.I., Clark, V.H.: CERR: A Computational Environment for Radiotherapy Research. Med. Phys. 30, 979-985 (2003)

10. Dias, J., Rocha, H., Ferreira, B.C., Lopes, M.C.: A genetic algorithm with neural network fitness function evaluation for IMRT beam angle optimization. Cent. Eur. J. Oper. Res. (in press) doi:10.1007/s10100-013-0289-4

11. Lee, E.K., Fox, T., Crocker, I.: Integer programming applied to intensity-modulated radiation therapy treatment planning. Ann. Oper. Res. 119, 165-181 (2003)

12. Li, Y., Yao, D., Yao, J., Chen, W.: A particle swarm optimization algorithm for beam angle selection in intensity modulated radiotherapy planning. Phys. Med. Biol. 50, 3491-3514 (2005)

13. Liu, H.H., Jauregui, M., Zhang, X., Wang, X., Dongand, L., Mohan, R.: Beam angle optimization and reduction for intensity-modulated radiation therapy of non-smallcell lung cancers. Int. J. Radiat. Oncol. Biol. Phys. 65, 561-572 (2006)

14. Pugachev, A., Xing, L.: Computer-assisted selection of coplanar beam orientations in intensity-modulated radiation therapy. Phys Med Biol. 46, 2467-2476 (2001)

15. Pugachev, A., Xing, L.: Incorporating prior knowledge into beam orientation optimization in IMRT. Int. J. Radiat. Oncol. Biol. Phys. 54, 1565-74 (2002)

16. Rocha, H., Dias, J.M., Ferreira, B.C., Lopes, M.C.: Incorporating Radial Basis Functions in Pattern Search Methods: Application to Beam Angle Optimization in Radiotherapy Treatment Planning. Proceedings of ICCSA 2012, Lecture Notes in Computer Science 7335, 1-16 (2012)

17. Rocha, H., Dias, J.M., Ferreira, B.C., Lopes, M.C.: Beam angle optimization for intensity-modulated radiation therapy using a guided pattern search method. Phys. Med. Biol. 58, 2939-2953 (2013)

18. Rocha, H., Dias, J.M., Ferreira, B.C., Lopes, M.C.: Selection of intensity modulated radiation therapy treatment beam directions using radial basis functions within a pattern search methods framework. J. Glob. Optim. 57, 1065-1089 (2013)

19. Rocha, H., Dias, J.M., Ferreira, B.C., Lopes, M.C.: Pattern search methods framework for beam angle optimization in radiotherapy design. Appl. Math. Comput. 219, 10853-10865 (2013) 
20. Romeijn, H.E., Ahuja, R.K., Dempsey, J.F., Kumar, A., Li, J.: A novel linear programming approach to fluence map optimization for intensity modulated radiation therapy treatment planing. Phys. Med. Biol. 48, 3521-3542 (2003)

21. Stein, J., Mohan, R., Wang, X.H., Bortfeld, T., Wu, Q., Preiser, K., Ling, C.C., Schlegel, W.: Number and orientation of beams in intensity-modulated radiation treatments. Med. Phys. 24, 149160 (1997) 